\title{
The effect of cooling press on the encapsulation properties of crystalline photovoltaic modules: residual stress and adhesion
}

\author{
Heng-Yu Li ${ }^{1}$, Laure-Emmanuelle Perret-Aebi ${ }^{1}$, Valentin Chapuis ${ }^{1}$, Christophe Ballif ${ }^{1}$ and Yun Luo ${ }^{2 *}$ \\ 1 École Polytechnique Fédérale de Lausanne (EPFL), Institute of Microengineering (IMT), Photovoltaics and Thin Film Electronics \\ Laboratory, Rue Breguet 2, 2000 Neuchâtel, Switzerland \\ 2 3S Swiss Solar Systems AG, Schachenweg 24, 3250 Lyss, Switzerland
}

\begin{abstract}
A high-quality encapsulation process is crucial to ensuring the performance and long-term reliability of photovoltaic (PV) modules. In crystalline Si technology-based modules, poly (ethylene-co-vinyl acetate) (EVA) is the most widely used PV encapsulant. Its encapsulation process is usually performed in a flat-bed vacuum bag laminator. In certain types of laminators, cooling press can be applied to the module cooling process after the module encapsulation, leading to a much higher cooling rate $\left(\sim 100^{\circ} \mathrm{C} / \mathrm{min}\right)$ than conventional natural cooling due to the application of water cooling circulation and mechanical pressure on the modules. In this work, the effect of the cooling press on the encapsulation properties of PV modules with EVA as the encapsulant are assessed on the aspects of residual stress in the modules, peeling strength between glass and EVA, and the resulting EVA gel content after encapsulation. The results show that the cooling press influences the encapsulation properties of PV modules. In particular by applying the cooling press after encapsulation, the residual normal stress in the Si solar cell in the encapsulated module after cooling can be reduced by as much as $22 \pm 2$ to $27 \pm 3 \%$ depending on the EVA gel content, whereas the peeling strength between front glass and EVA is increased by $\sim 10 \%$. This work should help the further optimization of PV module encapsulation processes aimed at improving module encapsulation quality. Copyright (C) 2013 John Wiley \& Sons, Ltd.
\end{abstract}

\section{KEYWORDS}

cooling press; photovoltaic module; EVA; encapsulation; adhesion; residual stress

${ }^{*}$ Correspondence

Yun Luo, PlusMat AG, Höheweg 55, 3054 Schüpfen, Switzerland.

E-mail: yun.luo@plusmat.ch

Received 14 December 2011; Revised 30 January 2013; Accepted 24 June 2013

\section{INTRODUCTION}

Field-deployed photovoltaic (PV) modules frequently face a combination of various natural stresses [1]. The reliability of PV modules plays a crucial role in determining the performance degradation and lifetime thus the cost per $\mathrm{kWh}$ of PV electricity. As a result, module reliability is the utmost guarantee for ensuring the role of photovoltaic technology as a key energy resource in the future. Generally, failed crystalline Silicon (c-Si) technology-based PV modules suffer mainly from the degradation of the encapsulation scheme, of interface between the encapsulant and the cells, of the electrical contacts, instead of the c-Si solar cells (i.e., the p-n junction), which usually have much longer lifetime. Therefore, the encapsulation quality of the PV modules, very often overlooked, is actually critical to photovoltaic technology.

A c-Si based PV module is typically composed of a front glass, stringed solar cells sandwiched between two layers of polymeric encapsulant, and a back protection layer of either glass or polymeric backsheet. One major function of the polymeric encapsulant is to bond all components into one piece and hence provide the structural stability of PV modules. Besides that, a qualified PV encapsulant should also exhibit satisfactory performances in terms of optical transparency (for front encapsulant), chemical stability, electrical insulation, thermal conductivity, matching of the thermal expansion coefficients, moisture barrier property, etc. [2]. Nowadays, the most widely used 
encapsulant is a statistical copolymer, Poly (ethylene-covinyl acetate) (EVA), occupying nearly $80 \%$ of the PV encapsulant market [3]. In the EVA formulated for PV, peroxides are added as curing agents to initiate the cross-linking reaction and to transform EVA into an elastomer [4-6]. The EVA encapsulation process of PV modules is usually performed in a flatbed vacuum bag laminator [7-9]. Figure1a shows module temperature/pressure evolution curves during a typical encapsulation process. The heating plate temperature is normally set at between 140 and $160{ }^{\circ} \mathrm{C}$. The encapsulation process includes four main steps: (i) preheating of PV modules on metal pins while vacuum is generated to evacuate potentially trapped air in the module layup; (ii) after the preheating, the pins are removed and the PV module is directly pressed ( 1 bar) onto the heating plate by a membrane. The resulting improved heat transfer allows the laminate temperature to rise rapidly to the desired curing temperature; (iii) the EVA curing process is initiated by the thermally-activated peroxides once the processing temperature is reached. The EVA curing continues until the desired EVA gel content is reached [10,11]; and (iv) the PV module is transferred out of the lamination chamber and the cooling process starts.

In normal laminators, the cooling process occurs naturally in ambient environment. To enhance the quality control and the production output of the PV modules, an additional step at the end of the process, the so-called 'cooling press' (CP), has been introduced [12]. In this process, the PV modules are transferred directly into the $\mathrm{CP}$ chamber after the curing step of the EVA. Therein, the cooling process of the module is accelerated by a water circulation cooling system $\left(20^{\circ} \mathrm{C}\right)$ embedded into the metallic plate underneath, whereas 1 bar mechanical pressure is applied over the whole module by the membrane above, as schematically illustrated in figure $1 \mathrm{~b}$. The unique features of the CP-assisted cooling compared with the conventional cooling process lie mainly in the following two aspects: (i). a higher and controlled module cooling rate of more than $100{ }^{\circ} \mathrm{C} / \mathrm{min}$; and (ii). application of a controlled 1 bar pressure throughout the module cooling process.

Usually, for polymer materials including composites, the cooling rate and pressure have strong influence on properties like density, crystallinity, hardness, residual stress, etc. [13-15]. Thus, it is hypothesized that the cooling press may also affect the properties of the cured

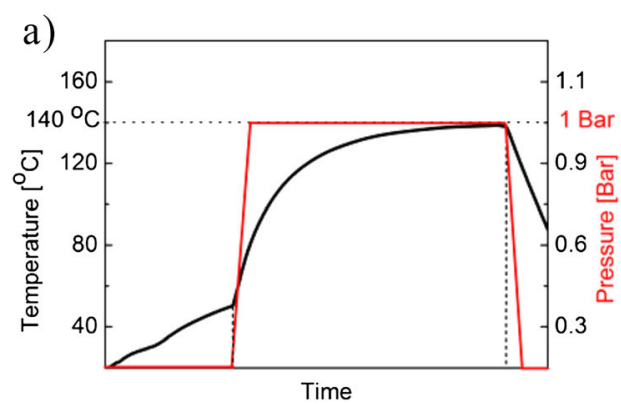

EVA encapsulant, and hence, the encapsulation properties of PV modules. In this work, the effect of cooling press is studied experimentally, with focus on the residual stress in the PV modules and the adhesion between EVA and glass after the encapsulation process.

\section{EXPERIMENTAL SETUP}

\subsection{Residual stress}

\subsubsection{Methodology.}

Several approaches have been proposed in the literature to study the residual stress in materials, especially layered composite materials [16-19]. PV modules, composed of different layers of materials, can be treated as a layered composite. However, the multilayered structure of PV modules makes it difficult to determine experimentally the quantitative distribution of residual stress across each layer. In this paper, a qualitative method is adopted, which we believe to be sufficient for the comparative evaluation of the effect of cooling press on the module encapsulation properties. Using the resulting curvature of the laminates to evaluate the residual stress is a common approach in the field of polymer composites [20,21]. In PV modules, due to the stiffness of the front glass, the overall module curvature induced by the residual stress is too small to be measured accurately. Inspired by the layer removal technique, commonly used to measure the stress distribution in many homogeneous material systems [17,22,23], a similar technique is deployed in this study. After the encapsulation, the front glass is removed from the rest of the module. Then the curvature of the laminate composed of remaining layers is measured to evaluate the residual stress.

\subsubsection{Sample preparation.}

Small modules of $200 \times 200 \mathrm{~mm}^{2}$ are encapsulated with the layups shown in Figure 2, either with (Figure 2a) or without cell (Figure 2b). The $156 \times 156 \mathrm{~mm}^{2}$ cells are placed right in the center. A Teflon sheet is inserted between the front glass and the top EVA layer, which allows an easy removal of front glass after encapsulation. The encapsulation is performed on a NG3622XLCP laminator equipped with a separate cooling chamber [10]. The curing b)

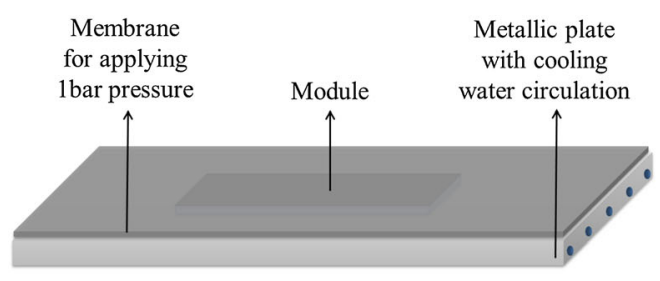

Figure 1. (a) Temperature (in black) and pressure (in red) profiles of a standard ethylene-vinyl acetate encapsulation process of photovoltaic modules, and (b) schematic drawing of the main components in the cooling press chamber. 
a)



c)

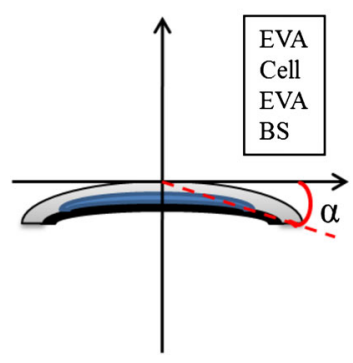

b)

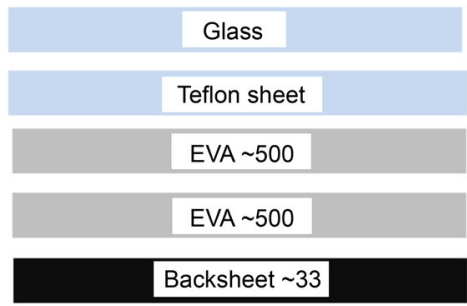

d)

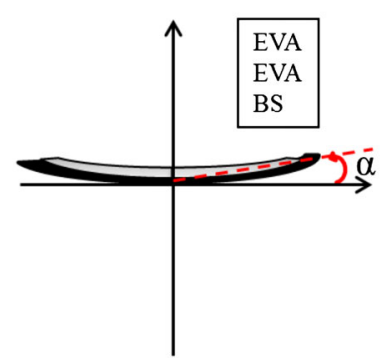

Figure 2. Layup of the sample modules prepared for the residual stress study: (a) module with one solar cell, (b) module without solar cell, (c) the curvature of the module with one cell after cooling, and (d) the curvature of the module without cell after cooling. In a and b, the numbers listed in the layers of ethylene-vinyl acetate cell and backsheet are their approximate thermal expansion coefficients, in $10^{-6} \mathrm{~K}^{-1}$. In $\mathrm{c}$ and $\mathrm{d}$, the angle $\alpha$ represents the magnitude of curvature of the module in this study.

temperature is fixed at $140{ }^{\circ} \mathrm{C}$. Modules with three different EVA gel contents are prepared by setting the curing time at 300,600, and $900 \mathrm{~s}$. After the curing step, the modules are either cooled naturally in ambient conditions or in the cooling chamber under cooling press. For each encapsulation condition, three modules are prepared, from which the average curvature is determined and reported later.

After the front glass removal, the laminates exhibit different curvatures depending on the module layup, as illustrated in Figures 2c and 2d. Experimentally, the resulting curvature is always found to be along one single axis, possibly due to the asymmetrical thermal shrinkage in certain module component. The magnitude of the curvature is represented by angle $\alpha$ in Figures $2 c$ and $d$. The maximum curvature angle $\alpha$ of each module is recorded in this work.

\subsection{Adhesion study}

\subsubsection{Sample preparation.}

The effect of cooling press on the adhesion between EVA and glass is studied on $670 \times 710 \mathrm{~mm}^{2}$ modules with layup similar to the one in Figure 2b. The difference is that, instead of complete coverage, the Teflon releasing sheets are inserted into the interface between EVA and glass only at five different locations on the module, functioning as initiators for the subsequent peeling test. Care is taken that the non-tin side of the glass is always laminated in contact with EVA [24]. The encapsulation process is identical to the one described in Section 2.1.2. One module is prepared for each encapsulation condition.

\subsubsection{Peeling test.}

The peeling test is performed by a handheld universal tester equipped with a load cell. The modules are measured in ambient environment after the encapsulation process (with or without cooling press) without any intentional accelerated aging. The peeling speed is controlled manually to be about $100 \mathrm{~mm} / \mathrm{min}$ for all tests. Near $180^{\circ}$ peeling test is carried out by using the backsheet/EVA composite as the peeling arm. After removing the pre-added Teflon sheets, the peeling is initiated thereby. Three peeling tests are performed at each of the five locations on every module. The average peeling strength of each tested module is reported in the adhesion study presented in the succeeding texts.

\subsubsection{Tensile test.}

In every module encapsulated for the adhesion study described in Section 2.2.1, an area of $100 \times 100 \mathrm{~mm}^{2}$ in the center is covered with two Teflon sheets, each below and above the EVA layer. This allows us to take out a piece of $100 \times 100 \mathrm{~mm}^{2}$ cured EVA for both the tensile test and the differential scanning calorimetry (DSC) study as described later. Therefore, the encapsulation process of EVA samples for the tensile test is identical to that described in Section 2.1.2.

The samples for tensile tests have a controlled geometry (cf. Figure 3) with an initial length between the machine arms $L_{0}=40 \mathrm{~mm}$, an initial thickness $t_{0}$ of $0.9-1.1 \mathrm{~mm}$ (as encapsulated) and an initial width $W_{0}=10 \mathrm{~mm}$. The width is reduced in the middle of the sample to about $6 \mathrm{~mm}$ to localize the tensile stress in this region. 


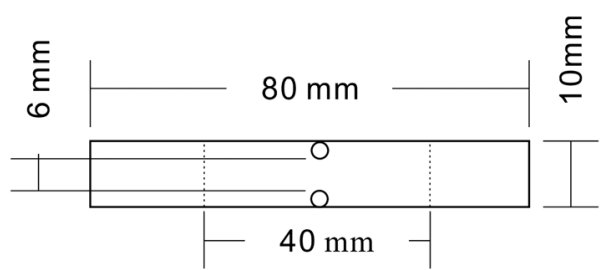

Figure 3. Geometry of the ethylene-vinyl acetate samples for the tensile testing.

The tensile tests are performed at $25^{\circ} \mathrm{C}$ in ambient environment on a Schenk universal tester equipped with a $2 \mathrm{kN}$ load cell. An arm displacement velocity of $100 \mathrm{~mm} / \mathrm{min}$ is used for all the samples. The characteristic of mechanical properties are calculated according to the ASTM D882 standard for tensile testing of polymeric sheets.

\subsection{Differential scanning calorimetry study}

Differential scanning calorimetry is used as a fast tool to evaluate the gel content and crystallization of cured EVA in this study. All EVA samples for the DSC study are cured as described in Section 2.2.3. The DSC analysis is performed by a Mettler Toledo DSC1 (Columbus, OH, USA). The DSC sample is prepared as a circular disc with a diameter of about $5 \mathrm{~mm}$ and a weight of about $10 \mathrm{mg}$ in an $\mathrm{Al}$ crucible. Thermograms are recorded under constant Nitrogen flow from 30 to $200^{\circ} \mathrm{C}$ at a heating rate of $10 \mathrm{~K} \mathrm{~min}^{-1}$.

\section{RESULTS AND DISCUSSIONS}

\subsection{Effect of cooling press on the residual stress}

The curing step of the PV module takes place at typically 140 to $160^{\circ} \mathrm{C}$. After the curing, the multilayered PV module cools down with or without cooling press, during which each component tends to shrink to an extent determined by its thermal expansion coefficient (CTE). However, as all the layers are bonded tightly after curing, the in-plane shrinkages are confined by the adhesive bonding between the neighboring layers. This inevitably results in thermal (residual) stresses built up in the module, leading to the commonly observed curvature of the modules after cooling.

Due to the mismatched CTE of different components, all tested modules exhibit certain curvature after cooling and the subsequent front glass removal, as shown in Figure 4d. The direction of the curvature depends on the actual layup. For the module without cell (Figure 2b), EVA has much larger CTE $\left(\sim 500 \times 10^{-6} \mathrm{~K}^{-1}\right)$ than that of the backsheet $\left(\sim 33 \times 10^{-6} \mathrm{~K}^{-1}\right)$, leading to a curvature toward the EVA layer. For the module with one cell (Figure 2a), the symmetrical structure of the EVA/cell/EVA layers and the sufficient bonding between the cell and EVA enable us to treat them as one piece with the effective thermal expansion coefficient close to the cell. In this
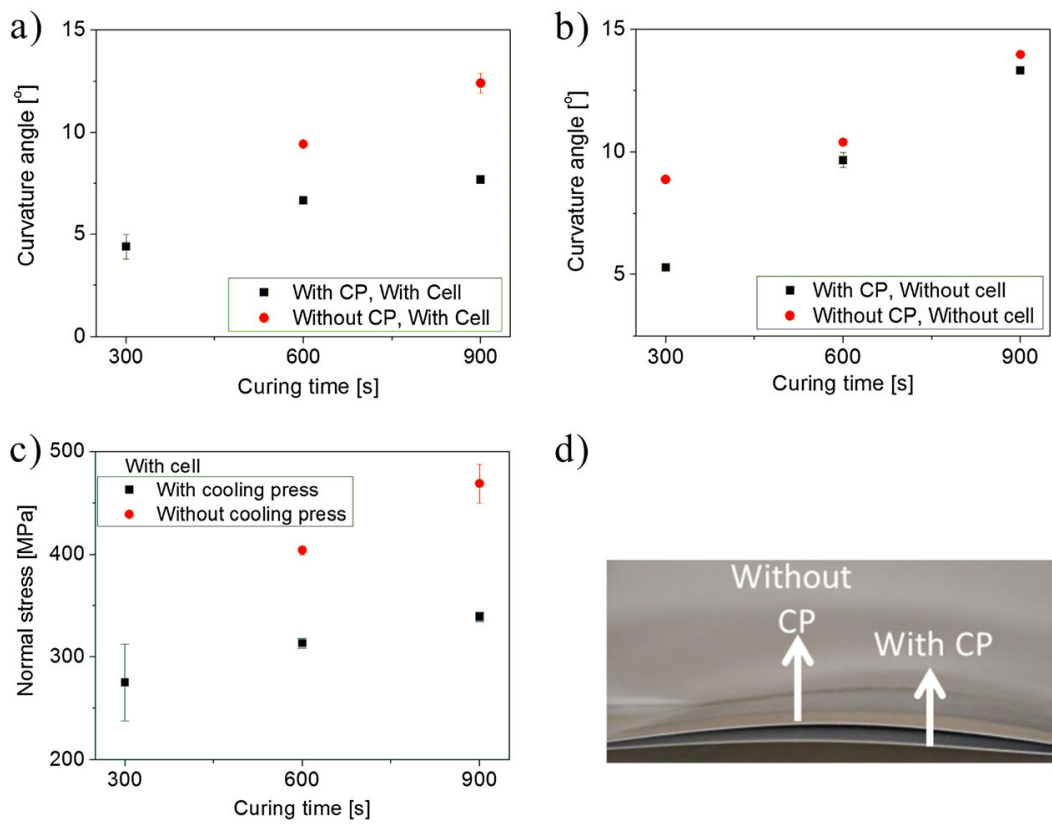

d)

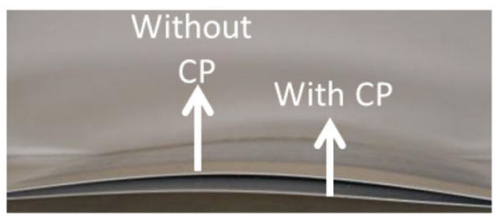

Figure 4. In $a$ and $b$, the curvature angles of the tested modules after cooling and front glass removal: (a) the modules with one cell, with or without cooling press (CP), (b) the modules without cell, with or without CP, (c) calculated normal stress at the middle layer of Si solar cell in the module with one cell, and (d) an image of two modules after cooling and front glass removal, with or without CP. Note: in a and $\mathrm{C}$, the data point for the module with cell and without $\mathrm{CP}$ is missing because of incapability of detaching the module from the release sheet without cell breakage. 
case, the whole module bends toward the backsheet which has a much larger CTE than the Si solar cell $\left(2.6 \times 10^{-6} \mathrm{~K}^{-1}\right)$.

The curvature angles resulting from different encapsulation conditions are summarized in Figure4. It shows the curvature angle $\alpha$ measured on the modules encapsulated with three different curing times, 300, 600, and 900 s, respectively. Figure 4 a shows the curvatures of the modules encapsulated with cells. Clearly the curvature increases with the curing time no matter whether the cooling press is applied after encapsulation or not. Figure $4 \mathrm{~b}$ shows the same trends for all the modules without cells. The EVA/ backsheet samples bending can be described using an elastic model for fast cooling of a simplified 2-layer system [25]. Eq. 1 describes the relationship between the curvature angle $\alpha$ and the residual stress $\sigma$ built in such a system.

$$
\alpha^{\propto} \frac{\sigma}{c_{1} E_{1}+c_{2} E_{2}}
$$

In which, $E_{1}$ and $E_{2}$ are the elastic modulus of two layers; $c_{1}$ and $c_{2}$ are constants depending on thicknesses and Poisson coefficients of the two layers. The stress being directly proportional to the measured curvature angle, one can conclude that a longer curing time causes higher residual stress in the studied 2-layer samples.

For the simplified 2-layer system, Eq. 2 states that the residual stress $\sigma$ depends on the polymer elastic modulus $E_{\mathrm{p}}$ and the potential strain $\varepsilon$ (including chemical and thermal strain). Like most thermoset polymers, EVA PV encapsulant experiences continuous chemical volume shrinkage during the curing process [19]. Moreover, the elastic modulus of the cured EVA encapsulant increases with its curing time [26]. According to Eq. 2, the larger chemical strain and higher elastic modulus result in higher residual stress built up in the longer-cured EVA [25].

$$
\sigma=-\frac{E_{p} \cdot \varepsilon}{\left(1-\gamma_{1}\right)}
$$

The case of the module with cell is more complex due to the presence of multiple layers of different materials. In order to evaluate the magnitude of residual stress in the Silicon solar cell inside the encapsulated module from the experimentally measured curvature angles, the theory for thermal bending of multilayers systems can be used. An extensive theoretical modeling on thermal stresses in a PV module has been presented recently in ref. [27]. Here a simplified approach based on the developments reported in [28] and [29] is used to calculate the normal stress in the different layers of a stack EVA/cell/EVA/backsheet that undergoes cooling from curing temperature $\left(140^{\circ} \mathrm{C}\right)$ to room temperature $\left(20^{\circ} \mathrm{C}\right)$. The details of the calculation are documented in the APPENDIX A. The results from the calculation are shown in Figure 4c, where the normal stress in the middle of the Si solar cell is obtained for different encapsulation conditions that as the curing time extends the stress in the solar cell increases greatly from 300 to 900 s curing time and cooled under pressure.

Now we discuss the effect of cooling press on the module residual stress. As illustrated in Figures $4 a$ and b, for modules with and without cell, the modules cooled under cooling press always show smaller curvature angle than the modules with the same curing time but without cooling press. In other words, the cooling press can lower the deflection of the laminates.

In Figure 4c, with the calculation approach described in the APPENDIX A, the reduction on the normal stress in the solar cell by the cooling press is estimated to be $\sim 22 \pm 2$ to $27 \pm 3 \%$, depending on the EVA gel content. The explanation of this effect of the cooling press remains open here for further study. However, it may be related to the different EVA chain rearrangement behaviors during the cooling with and without 1 bar compressive pressure, which could result in the different levels of EVA thermal shrinkage, and hence, the different residual stresses built up in the modules after cooling.

In the PV modules with the front glass, the curvature of the EVA/Cell/EVA/backsheet stack is suppressed by the bending momentum supplied by the glass. However, the built-in stress due to the differential thermal shrinkage of the EVA, cell and backsheet is still present and lower the additional external stress required to create defects such as delamination, cell breakage, etc [19]. Thus, decreasing the curvature angle of the module (without front glass) indicates the lowering of internal residual stress. The discussion earlier shows that the shear stress at the EVA/glass interface in the PV module can be partly reduced by applying the cooling press during cooling. The relieving effect of cooling press on the residual stress is expected to play a positive role on improving the PV module reliability.

\subsection{Effect of cooling press on adhesion}

The location-averaged peeling strengths of the modules with different encapsulation conditions are shown in Figure 5a. No matter whether the cooling press is applied, the measured peeling strength gradually decreases with the EVA curing time, i.e., from $\sim 217 \pm 10$ to $\sim 172 \pm 5 \mathrm{Ncm}^{-1}$. This result might be contradictory to the common sense about the influence of the curing time on the adhesion between glass and EVA in the PV module. To explain this phenomenon, one has to understand the physical meaning of the measured peeling strength.

During a peeling test, the total input energy generally consists of two terms: the elastic energy storage $\left(G_{\mathrm{e}}\right)$ and the plastic energy dissipation $\left(G_{\mathrm{p}}\right)$ in the peeling arm [30-32]. The $G_{\mathrm{e}}$ is elastically stored in the peel arm until delamination event occurs, whereas $G_{\mathrm{p}}$ is unrecoverable and cannot contribute to the delamination. Without considering the initial cracks that could influence the delamination, the delamination can be seen to only be initiated when $G_{\mathrm{e}}$ reaches a critical value $G_{\mathrm{a}}$, which corresponds to the interfacial adhesion fracture energy. The peeling strength normally reported in the literature actually reflects the total energy input $\left(G_{\mathrm{e}}+G_{\mathrm{p}}\right)$ in the peeling system. 



Figure 5. (a) The peeling strength measured on the modules encapsulated with and without cooling press for curing time of 300,600 , and $900 \mathrm{~s}$, (b) the tensile tests on the ethylene-vinyl acetate cured with different encapsulation processes. t300: the curing time of 300 s. WCP: with cooling press. WOCP: without cooling press.

Obviously, $G_{\mathrm{p}}$ has to be subtracted from the total energy input to obtain $G_{\mathrm{a}}$.

The magnitude of $G_{\mathrm{a}}$ can be obtained by combining multiple tensile tests and model fitting, as shown in ref [30]. Here a simplified approach is taken to further discuss the effect of curing time on the measured peeling strength shown in figure 5a. It is reasonable to assume that the $G_{\mathrm{e}}$ and $G_{\mathrm{p}}$ in the backsheet are similar in all the peeling tests. Thus, the tensile tests are only performed on the EVA film samples instead of EVA/backsheet laminate for this discussion. Figure $5 \mathrm{~b}$ shows the results of tensile tests on the EVA samples prepared with different encapsulation conditions. Therein, the different colors correspond to curing time of 300,600 , and $900 \mathrm{~s}$, respectively. The solid curves represent the samples cooled with cooling press, and the dashed curves represent the samples cooled without cooling press. The tensile curve is elevated gradually with the EVA curing time. Under a fixed stress, $2.5 \mathrm{MPa}$ for example in Figure 5b, different amounts of energy are absorbed into the EVA. The $G_{\mathrm{e}}+G_{\mathrm{p}}$ in the EVA can be evaluated by integrating the area under the strain curve up to a fixed stress value. As seen, apparently the $300 \mathrm{~s}$ cured EVA peeling arm absorbs more energy than $600 \mathrm{~s}$ cured one, which in turn absorbs more than $900 \mathrm{~s}$ cured one. In other words, the curing time or gel content of EVA strongly affects the $G_{\mathrm{e}}+G_{\mathrm{p}}$ in the EVA. Thus, the difference on the peeling strength may not reflect the difference on the actual interfacial adhesion strength. Experimentally, it is observed that the 300 s cured EVA exhibits stronger plastic deformation than $600 \mathrm{~s}$ and $900 \mathrm{~s}$ cured ones after the peeling test, that is very likely the lowlycured EVA can cause more plastic energy dissipation than highly-cured EVA. Therefore, the higher peeling strength measured on modules with shorter curing time cannot prove stronger adhesion strength between EVA and glass and may be explained by the increase of plastic energy dissipation in the EVA/backsheet peeling arm.

In Figure 5a, the black symbols represent the modules cooled with cooling press, and the red ones represent those cooled without cooling press. One can see that, for all three curing times, the cooling press increases the peeling strength between front glass and EVA considerably. To examine the effect of cooling press on the actual adhesion strength, tensile tests are performed on the EVA processed with and without cooling press. The results are shown in Figure $5 \mathrm{~b}$. It can be seen clearly that under the measurement accuracy, the influence of cooling press on the EVA tensile curves is trivial and can be neglected. This result indicates that the magnitude of the $G_{\mathrm{p}}$ in the EVA peeling arm is not affected by the application of cooling press, which allows us to directly translate the difference in the peeling strength (i.e., total input energy) into the difference in the adhesion strength (i.e., adhesion fracture energy $G_{a}$ ). More precisely, it shows that the cooling press may enhance the actual interfacial adhesion strength between EVA and glass in the PV modules under study. This effect can be understood by considering the further development of the adhesion bonding during the cooling under 1 bar pressing, because the pressing can indeed enhance the intimate physical contact between EVA and glass during the cooling. Besides that, the reduced residual stress by cooling press may result in less adhesion bonding loss due to the formation of micro failures in the cooling process than that without cooling press.

In practice, the delamination and subsequent void formation in the PV module depends not only on the adhesive fracture energy $\left(G_{\mathrm{a}}\right)$, but also on the mechanical property of the EVA layer, which is included in the information obtained from $G_{\mathrm{e}}$ and $G_{\mathrm{p}}$. The traditionally adopted term 'peeling strength' is actually a combinational measure of $G_{\mathrm{e}}$ and $G_{\mathrm{p}}$. Therefore, a future debate in the PV community is necessary on whether $G_{\mathrm{a}}$ or peeling strength is more appropriate to use when assessing the influence of encapsulation quality on the long-term reliability of PV modules.

\subsection{Effect of cooling press on the EVA gel content}

The cooling press accelerates the cooling rate of PV modules by at least a factor of two. Thus, it is expected that the crystallization in the cured EVA will be affected by the cooling press. Also as a result of the higher cooling rate, 

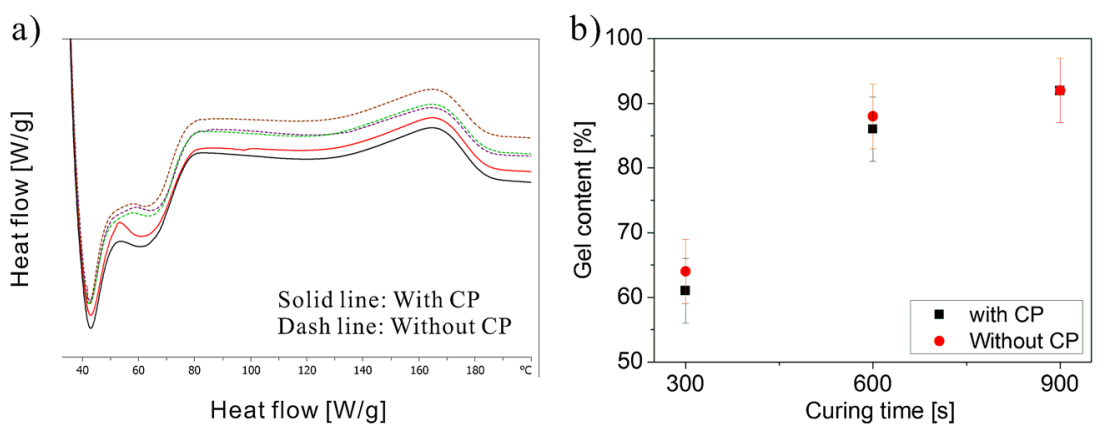

Figure 6. Differential Scanning Calorimetry (DSC) study on the effect of cooling press (CP). (a) the effect of CP on the crystallization in ethylene-vinyl acetate (EVA) with curing time of $300 \mathrm{~s}$. The solid curves represent EVA with cooling press, and the dash ones represent EVA without cooling press. Note: the layup of the curves in $y$-direction does not reflect any quantitative information, only for clear illustration purpose. (b) the effect of CP on the EVA gel content determined by DSC.

the modules with cooling press will stay above $120^{\circ} \mathrm{C}$ for shorter time than the naturally cooled modules after the encapsulation. As the cross-linking reaction can continue at the temperature above $120^{\circ} \mathrm{C}$, the EVA with natural cooling might experience longer post curing period than the one with cooling press. To study the effect of cooling press on the crystallization and the gel content of EVA, DSC is employed as the analytical tool [33].

Figure $6 \mathrm{a}$ shows the effect of the cooling press on the crystallization of the EVA cured for $300 \mathrm{~s}$. The solid curves correspond to two EVA samples taken from the modules with cooling press, whereas the dash curves corresponds to three EVA samples taken from the modules without cooling press. As seen, the cooling press clearly affects the profile of the $2^{\text {nd }}$ melting peak with maximum between 60 to $70^{\circ} \mathrm{C}$. For EVA with cooling press, the maximum peak position is $\sim 65^{\circ}$ $\mathrm{C}$, whereas it is $\sim 60^{\circ} \mathrm{C}$ for EVA without cooling press. The profile of the $2^{\text {nd }}$ melting peak reflects the size distribution of the crystallites in EVA after cooling [33]. The small crystallites have lower melting point than the big ones. Therefore, the observed influence can be explained by the different cooling rates due to the application of cooling press. By cooling press, the cooling rate is increased, which hinders the growth of the crystalline phase in the cured EVA and results in smaller crystallites.

Differential scanning calorimetry is a proven tool for fast determination on the gel content of EVA for PV application [34]. Figure $6 \mathrm{~b}$ shows the effect of cooling press on the gel content of EVA cured for 300,600, and 900 s. Here the EVA gel content is determined by quantifying the enthalpy of the broad exothermic peak in the DSC thermography, using the methodology described in ref [34]. As one can see, the difference in the gel content caused by the cooling press is within the measurement error, almost negligible for all cases. On the basis of our previous DSC and DMA study on the evolution of EVA gel content with different curing time (not shown here), the development of the EVA gel content slows down gradually as the curing time develops [26,34]. This explains why the longer post curing time in the natural cooling process does not increase the EVA gel content significantly.

\section{CONCLUSION}

The effect of cooling press on the encapsulation properties of crystalline photovoltaic modules is assessed primarily in terms of the residual stress and the adhesion between EVA and glass. The cooling press is shown to reduce the residual stress in the PV modules after the encapsulation process. The results also indicate that the cooling press can improve the adhesion between EVA and glass in the PV modules. The DSC study shows that the cooling press has only negligible influence on the EVA gel content in the PV modules. However, the cooling press does hinder the crystal growth in the EVA after the encapsulation. The curing time is also shown to affect the encapsulation properties of crystalline PV modules. The longer curing time results in higher residual stress in the modules and lower peeling strength between glass and EVA after encapsulation. This work shows that the cooling press applied after the EVA curing step considerably modifies the encapsulation properties of the c-Si based PV modules on the aspects of the residual stress and the EVA/glass adhesion after the encapsulation process. This conclusion is of great value on further understanding and optimizing the manufacturing process of c-Si PV modules with EVA encapsulants.

\section{ACKNOWLEDGEMENTS}

The authors are grateful for continuous financial support of this project from 3S Modultec, Switzerland and the Swiss Federal Office for Energy under project 101191. The authors also thank the partial support from the Archinsolar project financed by Swiss Electric Research (SER), the Swiss Federal Office for Energy (SFOE), the Competence Center Energy and Mobility (CCEM) and the Services Industriels Genevois (SIG). 


\section{REFERENCES}

1. Czanderna AW, Pern FJ. Encapsulation of PV modules using ethylene vinyl acetate copolymer as a pottant: a critical review. Solar Energy Materials and Solar cells 1996; 43: 101-181.

2. Lewis KJ. Encapsulant material requirements for photovoltaic modules. Polymers in Solar Energy Utilization June 15, 1983: 367-385.

3. GBI Research. Ethylene vinyl acetate (EVA) global market to 2015 - photovoltaic encapsulants to drive EVA demand in the future. Report 2011.

4. Pern FJ. Ethylene-vinyl acetate (EVA) encapsulants for photovoltaic modules: degradation and discoloration mechanisms and formulation modifications for improved photostability. Angewandte Makromolekulare Chemie 1997; 252: 195-216.

5. Pern FJ and Czanderna AW. Characterization of ethylene vinyl acetate (EVA) encapsulant: effects of thermal processing and weathering degradation on its discoloration. Solar Energy Materials and Solar Cells 1992; 25: 3-23.

6. Hidalgo M, Medlege F, Vite M, Corfias-Zuccalli C, Voarino P, González-León J. A new DSC method for the quality control of PV modules:simple and quick determination of the degree of crosslinking of EVA encapsulants. Photovoltaics International journal 2011; November issue: 130-140.

7. El Amrani A, Mahrane A, Moussa FY, Boukennous Y. Solar module fabrication. International Journal of Photoenergy 2007; Article ID 27610. DOI: 10.1155/ 2007/27610

8. Zahnd J, Boos C. Machine for the production of sheet elements from composite material. WO2006128699.

9. Lange RFM, Luo Y, Polo R, Zahnd J. The lamination of photo-voltaic modules. InterPV Asia 2009; June issue: 8 .

10. Luo Y, Fidalgo I, Lange RFM. Reducing encapsulation cycle time in PV module production. Global Solar Technology 2010; March issue: 14-16.

11. Lange RFM, Luo Y, Polo R, Zahnd J. The lamination of (multi)crystalline and thin film based photovoltaic modules. Progress in Photovoltaics: Research and Applications 2011; 19: 127-133.

12. The cooling press of the processed laminate was introduced by $3 \mathrm{~S}$ in 2001.

13. Scharnowski D. Characterisation of the influence of cooling rates on structure and properties of dynamic vulcanizates. Doctoral Dissertation 2005. MLU Halle-Wittenberg.

14. La Carrubba V, Brucato V, Piccarolo S. Influence of 'controlled processing conditions' on the solidification of iPP, PET and PA6. Macromolecular Symposia 2002; 180: 43-60.
15. Parlevliet PP. Residual strains in thick thermoplastic composites: an experimental approach. TU Delft. ISBN: 978-94-6113-0051.

16. Schajer GS. Relaxation methods for measuring residual stresses: techniques and opportunities. Experimental Mechanics 2010; 50: 1117-1127.

17. Treuting RG, Read WT, Jr. A mechanical determination of biaxial residual stress in sheet materials. Journal of Applied Physics 1951; 22: 130-134.

18. Eijpe MPIM, Powell PC. Residual stress evaluation in composites using a modified layer removal method. Composite Structures 1997; 37: 335-342.

19. Isaac RV. Residual stresses in composites. In Advanced Topics in Characterization of Composites. Trafford Publishing: Bloomington, Indiana, USA, 2004.

20. Kim KS, Hahn HT. Residual stress development during processing of graphite/epoxy composites. Composites Science and Technology 1989; 36: 121-132.

21. Coxon LD, White JR. Measurement of internal stresses in chemically cross-linked high-density polyethylene. Journal of Materials Science 1979; 14: 1114-1120.

22. Paterson MWA, White JR. Layer removal analysis of residual stress Part 2 a new procedure for polymer mouldings with depth-varying Young's modulus. Journal of Materials Science 1989; 24: 3521-3528.

23. Wagner AH, Yu JS, Kalyon DM. Orientation and residual stress distributions in injection molded engineering plastics. ANTEC '89: Plastics Create a World of Difference; New York, USA 1989: 303-307.

24. Yang B, Townsend PD, et al. Cathodoluminescence and depth profiles of tin in float glass. Journal of Physics D: Applied Physics 1994; 27: 1757-1762.

25. Ramani K, Zhao W. The evolution of residual stresses in thermoplastic bonding to metals. International Journal of Adhesion and Adhesives 1997; 17: 353-357.

26. Unpublished results from our Dynamical Mechanical Analysis (DMA) study on the EVA encapsulant.

27. Bechou L, Shangguan D, Suhir E. Predicted thermal stresses in a photovoltaic module (PVM). Photovoltaics International 16th Edition 2012: 118-130.

28. Hsueh CH. Thermal stresses in elastic multilayer systems. Thin Solid Films 2002; 418: 182-188

29. Zhang N-H. Thermoelastic stresses in multilayered beams. Thin Solid Films 2007; 515: 8402-8406.

30. Kawashita LF, Moore DR, Williams JG. Protocols for the measurement of adhesive fracture toughness by peel tests. The Journal of Adhesion 2006; 82: 10,973-995.

31. Moore DR. An introduction to the special issue on peel testing. International Journal of Adhesion and Adhesives 2008; 28: 153-157.

32. Kinloch AJ, Lau CC, Williams JR. The peeling of flexible laminates. International Journal of Fracture 1994; 66: 45-70. 
33. Li HY, Perret-Aebi LE, Theron R, Ballif C, Luo Y, Lange RFM. Towards in-line determination of EVA Gel content during PV modules lamination processes. Proceedings of 25th EU PVSEC 2010: 4044-4046.

34. Li HY, Perret-Aebi LE, Théron R, Ballif C, Luo Y, Lange RFM. Optical transmission as a fast and non-destructive tool for determination of ethylene-co-vinyl acetate curing state in photovoltaic modules. Progress in Photovoltaics: Research and Applications 2013; 21: 187-194.

\section{APPENDIX A}

Thermal bending of multilayers systems is a welldocumented problem [1-3]. Usually, the expressions are derived directly from the elastic beam bending theory [4]. We present here a simplified approach based on the developments reported in [1] and [2] to calculate the normal stress in the different layers of a stack of EVA/cell/EVA/ backsheet that undergoes cooling from lamination temperature $\left(140^{\circ} \mathrm{C}\right)$ to room temperature $\left(20^{\circ} \mathrm{C}\right)$.

Let's assume that we have a thin sandwich structure (in-plane directions $\mathrm{X}$ and $\mathrm{Y}$ infinite as compared with thickness direction $Z$ ) composed of $N$ layers with elastic

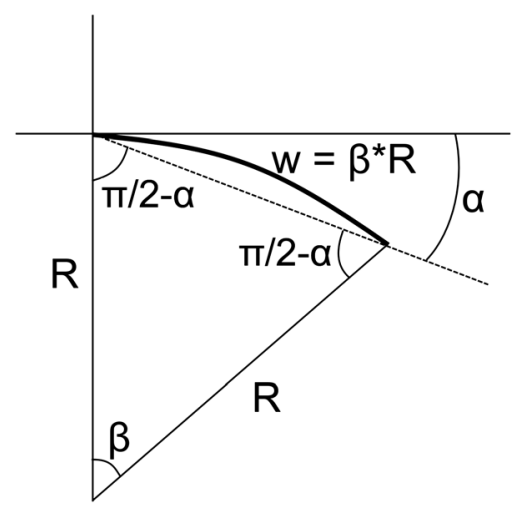

Figure A1. The geometrical analysis for the conversion from the curvature angle $\alpha$ to the curvature radius $R$. modulus $E_{j}$, thermal coefficients $\alpha_{j}$ and thicknesses $t_{j}$, free of stress at $T 0$. Increasing or decreasing the temperature of this structure by $\Delta T$ will lead to different thermal dilatation/contractions for each layers resulting in net normal stresses in each layer, as expressed in equation (1), in the case of uniaxial bending.

$$
\sigma_{j}(\Delta, T, z)=E_{j} \Delta T \cdot\left(\frac{\sum_{m=1}^{N} E_{m} \alpha_{m} t_{m}}{\sum_{m=1}^{N} E_{m} t_{m}}-\alpha_{j}\right)+\frac{E_{j}(z-\tau)}{R}
$$

$\sigma_{\mathrm{j}}$ in equation (1) gives the thickness dependence of the normal stress in layer $j$ for a temperature difference $\Delta T$ as compared with the stress-free state. In this equation, $z$ represents the thickness coordinate, $R$ represents the curvature radius of the sandwich and $\tau$ is defined as (2):

$$
\tau=\frac{t}{2}+\frac{1}{2} \frac{\sum_{m=1}^{N} \sum_{k=1}^{N} E_{m} t_{m} \beta_{m k} t_{k}}{\sum_{m=1}^{N} E_{m} t_{m}}
$$

In equation (2), $t$ represents the total thickness of the sandwich and $\beta_{j k}$ is a coefficient given by (3):

$$
\beta_{j k}=\left\{\begin{array}{c}
1, j>k \\
0, j=k \\
-1, j<k
\end{array}\right.
$$

In our case, the radius of curvature $R$ in (1) can be calculated from the measured deflection angles $\alpha$ of the laminates after cooling (see $\$ 2.1 .2$ of the manuscript) using a simple geometric analysis, as shown in figure A1.

$$
R=\frac{w}{2 \cdot \alpha}
$$

Where $w$ represents half of the (square) cell dimension, which was $156 \mathrm{~mm}$ in our study.

Table A1. Materials properties used in the model for normal stress calculation.

\begin{tabular}{lcccl}
\hline Material & Elastic modulus $[\mathrm{Pa}]$ & Thermal coefficient $[-]$ & Thickness $[\mathrm{mm}]$ & Source \\
\hline Silicon solar cell & $160 \times 10^{9}$ & $2.6 \times 10^{-6}$ & 0.2 & From ref [5] \\
TPT backsheet & $24 \times 10^{9}$ & $33 \times 10^{-6}$ & 0.35 & From ref [5] \\
EVA (uncured) & $5 \times 10^{6}$ & $15 \times 10^{-4}$ & 0.47 & Manufacturer's datasheet \\
EVA (63\% gel) & $9.4 \times 10^{6}$ & $8.1 \times 10^{-4}$ & 0.47 & Mixture law \\
EVA (87\% gel) & $11.1 \times 10^{6}$ & $5.4 \times 10^{-4}$ & 0.47 & Mixture law \\
EVA (93\% gel) & $11.5 \times 10^{6}$ & $4.8 \times 10^{-4}$ & 0.47 & Mixture law \\
EVA (fully cured) & $12 \times 10^{6}$ & $4 \times 10^{-4}$ & 0.47 & Manufacturer's datasheet \\
\hline
\end{tabular}

Fixing $T=120^{\circ} \mathrm{C}$, which corresponds to a cooling from ethylene-vinyl acetate (EVA) lamination temperature $\left(140^{\circ} \mathrm{C}\right)$ to room temperature and using both equation (4) to calculate curvature radius from measured deflections and table I for materials properties, we are able to calculate the residual stress in the solar cell. For comparison of the different conditions (different curing times and usage or not of the cooling press), we decided to calculate the normal stress in the middle of the solar cell thickness. Figure 8 shows the results of the stress analysis. 


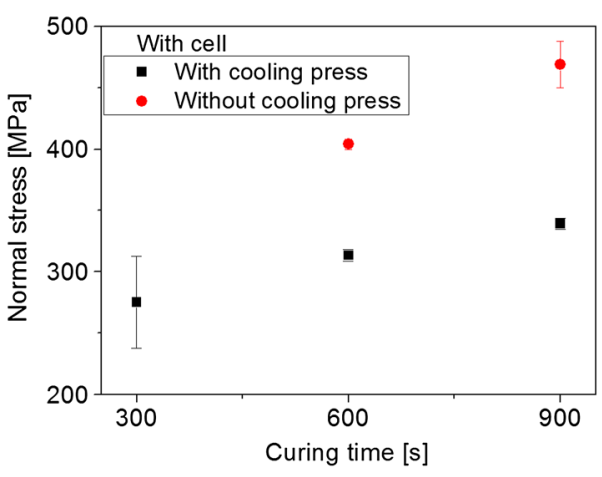

Figure A2. Effect of the curing time (gel content) and cooling press on residual normal stress in the solar cell.

To calculate the stresses using (1) and (4), we still need to know the different materials properties (elastic modulus and thermal expansion coefficient) and thicknesses. Table AI summarizes the numbers used in this study. Material properties for the considered EVA (gel content of 63,87 , and $93 \%$ corresponding to curing times of 300 , 600 , and $900 \mathrm{~s}$ ) have been calculated from properties of uncured and fully cured EVA using a simple rule of mixture.

Clearly, the stress levels shown in figure A2 strongly depend on the material properties (elastic modulus and thermal expansion coefficients) and on geometrical parameters (thickness of the layers). In the case of the present example, the accurate materials properties are not accessible and we have adopted values from different sources as listed in table I. The layers thicknesses were not measured after lamination but were taken as the initial values before lamination. This of course does not affect the cell and backsheet material, but small variation in the EVA thickness may occur. For those reasons, the authors would draw attention to the fact that the absolute calculated stress values may not be equivalent to the actual stresses. However, we notice that the calculated values are on the same order of magnitude as those obtained in [5]. The major effect that we want to demonstrate with this analysis is the strong influence on the residual stress by the cooling press and EVA gel content.

[1] Hsueh CH. Thermal stresses in elastic multilayer systems. Thin Solid Films 418 2002: 182-188.

[2] Zhang NH. Thermoelastic stresses in multilayered beams. Thin Solid Films 515 2007: 8402-8406.

[3] Zhang XC, Xu BS, Wang HD, Wu YX. An analytical model for predicting thermal residual stresses in multilayer coating systems. Thin Solid Films 488 2005: 274-282.

[4] Timoshenko S. Analysis of bimetal thermostats. JOSA 11 1925: 233-255.

[5] Bechou L, Shangguan D, Suhir E. Predicted thermal stresses in a photovoltaic module (PVM). Photovoltaics International 16th Edition 2012: 118-130. 